\title{
Electric bus fleet size and mix problem with optimization of charging infrastructure
}

Rogge, Matthias; van der Hurk, Evelien; Larsen, Allan; Sauer, Dirk Uwe

\section{Published in:}

Applied Energy

Link to article, DOI:

10.1016/j.apenergy.2017.11.051

Publication date:

2018

Document Version

Peer reviewed version

Link back to DTU Orbit

Citation (APA):

Rogge, M., van der Hurk, E., Larsen, A., \& Sauer, D. U. (2018). Electric bus fleet size and mix problem with optimization of charging infrastructure. Applied Energy, 211, 282-295.

https://doi.org/10.1016/j.apenergy.2017.11.051

\section{General rights}

Copyright and moral rights for the publications made accessible in the public portal are retained by the authors and/or other copyright owners and it is a condition of accessing publications that users recognise and abide by the legal requirements associated with these rights.

- Users may download and print one copy of any publication from the public portal for the purpose of private study or research.

- You may not further distribute the material or use it for any profit-making activity or commercial gain

- You may freely distribute the URL identifying the publication in the public portal

If you believe that this document breaches copyright please contact us providing details, and we will remove access to the work immediately and investigate your claim. 


\title{
Electric bus fleet size and mix problem with optimization of charging infrastructure
}

\author{
Matthias Rogge ${ }^{\mathrm{a}, \mathrm{b}, *}$, Evelien van der Hurk ${ }^{\mathrm{c}}$, Allan Larsen ${ }^{\mathrm{c}}$, Dirk Uwe Sauer ${ }^{\mathrm{a}, \mathrm{b}, \mathrm{d}}$ \\ a Chair for Electrochemical Energy Conversion and Storage Systems, Institute for Power Electronics and Electrical Drives (ISEA), RWTH Aachen University, Jaegerstrasse \\ 17-19, 52066 Aachen, Germany \\ b Juelich Aachen Research Alliance, JARA-Energy, Germany \\ ${ }^{\mathrm{c}}$ DTU Management Engineering, Technical University of Denmark, Denmark \\ d Institute for Power Generation and Storage Systems (PGS), E.ON Energy Research Center, RWTH Aachen University, Germany
}

\section{H I G H L I G H T S}

- A framework for the cost-efficient planning of battery bus fleets is proposed.

- The approach combines a genetic algorithm and mixed-integer-linearprogramming.

- Two electrification scenarios of European cities are analyzed in a case study.

- Energy efficiency is discussed for two competing battery bus concepts.

- Operation of lightweight buses enables energy savings of about 30\%.

\section{A R T I C L E I N F O}

\section{Keywords:}

Electric bus scheduling

Charger scheduling

Transportation system modeling

Infrastructure planning

TCO optimization

Genetic algorithm

\section{G R A P H I C A L A B S T R A C T}

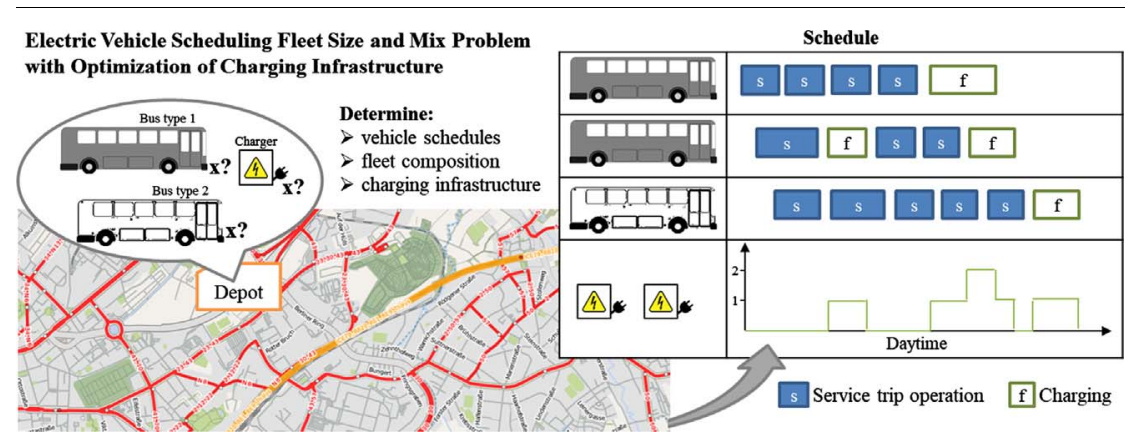

\begin{abstract}
A B S T R A C T
Battery electric buses are seen as a well-suited technology for the electrification of road-based public transport. However, the transition process from conventional diesel to electric buses faces major hurdles caused by range limitations and required charging times of battery buses. This work addresses these constraints and provides a methodology for the cost-optimized planning of depot charging battery bus fleets and their corresponding charging infrastructure. The defined problem covers the scheduling of battery buses, the fleet composition, and the optimization of charging infrastructure in a joint process. Vehicle schedule adjustments are monetized and evaluated together with the investment and operational costs of the bus system. The resulting total cost of ownership enables a comparison of technical alternatives on a system level, which makes this approach especially promising for feasibility studies comprising a wide range of technical concepts. Two scenarios of European cities are analyzed and discussed in a case study, revealing that the cost structure is influenced significantly by the considered bus type and its technical specifications. For example, the total energy consumption of the considered lightweight bus is up to $32 \%$ lower than the total consumption of the high range bus, although the deadheading mileage increases. However, the total costs of ownership for operating both bus types are relatively close, due to the increased fleet size and driver expenses required for the lightweight bus system. The case study furthermore reveals that a mixed fleet of different bus types could be advantageous depending on the operational characteristics of the bus route.
\end{abstract}

\footnotetext{
* Corresponding author at: Chair for Electrochemical Energy Conversion and Storage Systems, Institute for Power Electronics and Electrical Drives (ISEA), RWTH Aachen University, Jaegerstrasse 17-19, 52066 Aachen, Germany.

E-mail address: batteries@isea.rwth-aachen.de (M. Rogge).
} 


\begin{tabular}{|c|c|c|c|}
\hline \multicolumn{2}{|c|}{ Nomenclature } & \multirow{2}{*}{$\begin{array}{l}p \\
r^{k} \\
S\end{array}$} & \multirow{2}{*}{$\begin{array}{l}\text { purchasing costs per charger in } € \\
\text { recharging capability of bus type } k \in V \\
\text { set of nodes representing } n \text { service trips }\end{array}$} \\
\hline$A$ & set of arcs, union of $A^{s}$ and $A^{f}$ & & \\
\hline$A^{f}$ & set of arcs connecting charging events by charger & $S_{0}$ & set of nodes, union of $S$ and $0_{s}$ \\
\hline$A^{s}$ & set of arcs representing feasible deadhead trips & $S_{n+1}$ & set of nodes, union of $S$ and $q_{S}$ \\
\hline$a_{i}$ & starting time of event $i \in S \cup F$ & $t_{i}$ & duration of service trip $i \in S$ in seconds \\
\hline$c_{e}^{k}$ & energy costs per unit for bus type $k \in V$ & $t_{i j}$ & time required for servicing trip $j \in S_{n+1}$ after trip $i \in S_{0}$ in \\
\hline$c_{t}$ & time related operational costs for a bus in $€$ per hour & & seconds \\
\hline $\begin{array}{l}d_{f} \\
E^{k}\end{array}$ & $\begin{array}{l}\text { duration of charging event } f \in F \text { in seconds } \\
\text { usable battery capacity of bus type } k \in V\end{array}$ & $u_{f}$ & $\begin{array}{l}\text { supplement for postponing charging event } f \in F \text { in sec- } \\
\text { onds }\end{array}$ \\
\hline$e_{i}^{k}$ & $\begin{array}{l}\text { current energy level of a bus of type } k \in V \text { after servicing } \\
\text { trip } i \in S_{n+1}\end{array}$ & $\begin{array}{l}V \\
w\end{array}$ & $\begin{array}{l}\text { set of bus types, type } k \in V \\
\text { weighting factor for shift penalties between } 0 \text { and } 1\end{array}$ \\
\hline $\begin{array}{l}F \\
F_{0}\end{array}$ & $\begin{array}{l}\text { set of nodes representing possible charging events } f \in F \\
\text { union nodes representing all charging events } F \text { and the }\end{array}$ & $x_{i j}^{k}$ & $\begin{array}{l}\text { binary indicator whether bus type } k \text { services event } j \text { after } \\
\text { event } i, k \in V,(i, j) \in A^{s}\end{array}$ \\
\hline$F_{n+1}$ & $\begin{array}{l}\text { bus depot source node } 0_{f} \\
\text { union nodes representing all charging events } F \text { and the }\end{array}$ & $z_{l m}$ & $\begin{array}{l}\text { binary indicator of charging event } m \text { takes place after } \\
\text { charging event } l,(l, m) \in A^{f}\end{array}$ \\
\hline$G$ & $\begin{array}{l}\text { bus depot sink node } q_{f} \\
\text { multi-graph defined by node set } N \text { and arc set } A\end{array}$ & $0_{f}, q_{f}$ & $\begin{array}{l}\text { source and sink node representing bus depot for charging } \\
\text { events }\end{array}$ \\
\hline$h_{i j}^{k}$ & $\begin{array}{l}\text { energy consumption for servicing trip } j \in N_{n+1} \text { after trip } \\
i \in N_{0} \text { using vehicle type } k \in V \text { in } \mathrm{kWh}\end{array}$ & $0_{s}, q_{s}$ & $\begin{array}{l}\text { source and sink node representing bus depot for service } \\
\text { trips }\end{array}$ \\
\hline$M$ & Constant, $M \gg a_{i}$ & $\beta_{i}$ & start time of service trip $i \in S$ \\
\hline$m^{k}$ & Purchasing costs of bus type $k \in V$ in $€$ & $\gamma$ & earliest start time of charging events \\
\hline$N$ & Set of nodes, union of $F, 0_{f}, q_{f}, \mathrm{~S}, 0_{s}$ and $q_{s}$ & $\delta$ & latest feasible start time of charging events \\
\hline
\end{tabular}

\section{Introduction}

Emission mitigation is one of the major topics of the 21st century. Negative consequences of the continuously increasing output can be observed on the global and local scales. Reducing the usage of fossil fuels is a commonly agreed upon measure to target this issue. The transport sector is requested to contribute by increasing the efficiency of conventional fuel-powered drivetrains and by introducing electric vehicle concepts powered by renewable energy sources [1-3]. Several national policies and subsidy schemes exist to promote this transition process $[4,5]$.

Especially commercial fleets, such as public transport buses, are seen as a prime starting point for the introduction of electric vehicles. Their operation is planned in advance and dominated by high mileages per vehicle, so that higher investment costs of the electric drivetrain could be compensated by reduced operational costs. Indeed, battery electric buses have been successfully tested in several projects worldwide [6] and, with decreasing battery system costs, have become increasingly competitive with conventional buses [7]. However, the reduced operational performance of electric buses is still a major barrier for the transition process. The aim of the present work is to contribute to this process by providing a framework for the cost-optimized planning of electric public transport bus fleets.

The paper addresses strategic electric bus planning by focusing on the "Electric Vehicle Scheduling Fleet Size and Mix Problem with Optimization of Charging Infrastructure" (EVS-FMC), minimizing the total cost of ownership (TCO) of electric vehicle fleets. The TCO is the main decision criterion for investment alternatives. It consists of the initial investments in vehicles and charging infrastructure, as well as the operational costs within a defined time period. Provided a set of service trips and a candidate set of vehicle types, the EVS-FMC proposes a fleet-composition investment, in terms of number of vehicles to by per vehicle type, as well as a vehicle schedule that serves all service trips, and a set of chargers to buy per depot, that all together minimize TCO.

The EVS-FMC can be considered as an extension of the "Vehicle Scheduling - Fleet Size and Mix Problem", with the addition of range constraints per vehicle, the scheduling of charging time, and the scheduling of charging infrastructure. It relates directly to the general "Fleet Size and Mix Problem" analyzed in operations research, and is a subcategory of the "Vehicle Routing Problem", in which routing is performed jointly with a determination of the required number of vehicles [8]. Fleets can consist of single vehicle types (homogeneous fleet) or multiple vehicle types (heterogeneous fleet). Recent work has mainly referred to the area of goods distribution. A comprehensive review focusing on electric vehicles in this field is provided in [9]. The discussed approaches differ in terms of the considered vehicle types (homogeneous or heterogeneous electric vehicle fleet, with and without conventional combustion engine vehicles) and the methodology of handling charging events. Goeke et al. and Lebeau et al. extensively studied the routing of mixed fleets composed of conventional and electric vehicles $[10,11]$. They emphasized the need to consider vehicle types' specific energy consumptions, especially for vehicles with varying weights; this motivates the energy consumption simulation in this work. Van Duin et al. did not consider battery charging [12], whereas Gonçalves et al. defined that charging could take place at every customer's location [13]. In another study, Hiermann et al. included an insertion of charging events so that vehicles could explicitly drive to recharging stations when needed [14]. However, none of these authors considered investments in charging infrastructure or usage fees. Sassi et al. included usage fees as time-dependent charging costs for a "Mixed Fleet Routing Problem" [15]. However, the number of chargers in their model was still pre-defined and fixed.

By focusing on public transport buses, the routing problem becomes a scheduling problem, because service trips (regular operation in passenger service) are fixed in time and location. Routing alternatives are limited to deadhead trips, which are the connecting elements between service trips. The electric vehicle scheduling problem can be seen as a "Vehicle Scheduling Problem with Route and Fueling Time Constraints". The objective is to minimize fleet size and operational expenses. As for vehicle routing, previous studies differ in their way of considering battery charging. Li proposed a methodology for scheduling a fleet of battery buses with battery renewal or fast charging [16]. The implemented truncated column generation with variable fixing and local search is highly competitive, but the durations of charging events are not linked to the energy consumption, and charging costs are defined as a fixed value per event. Other approaches considering the charging in more detail are based, for example, on ant colony optimization $[17,18]$. However, these approaches do not provide the ability to handle heterogeneous fleets. In contrast, Paul and Yamada focused on the problem of fast-charging bus operation and scheduling charging 
events [19]. However, their implemented k-greedy algorithm can be seen as a drawback in terms of solution quality, because it does not focus on a global optimum. In another study, Chao et al. set up a multiobjective optimization for the scheduling of battery exchange buses operated in Shanghai [20]. Their approach aims to minimize the fleet size and the number of standby batteries, which are both related to investment costs, as well as the grid load. Their considerations are limited to homogeneous fleets. Ke et al. introduced the scheduling of battery buses, the handling of charging events, and the consideration of entire system costs [21]. They analyzed a suburban bus network in Taiwan and utilized a scheduling approach based on a sequential process. However, in this approach the assignment of bus types to routes is pre-defined, and the number of chargers always equals the number of buses, which is an overestimation. Wen et al. developed an adaptive large neighborhood search to minimize investment and operational expenses of homogeneous electric vehicle fleets [22]. The approach serves to analyze the consequences of locating charging stations in an artificial dataset. However, the authors do not consider investments in charging infrastructure.

In the operations research discipline, further studies have focused especially on the costs and environmental benefits of an electrification. Conclusions regarding the fleet mix are based on the calculated performance of alternative drive trains with standardized test cycles [23] or real-world operations data [24]. Other publications have especially emphasized the design and sizing of the technical components, without considering vehicle schedule adjustments [25-28]. In their work, Olsson et al. addressed the strong linkage between vehicle scheduling and technical planning [29]. They described the necessity to adjust the fleet size and to integrate charging phases in the schedule of depot charging battery buses. However, their work only introduced the problem without providing a solution approach.

The current literature is often specialized in a specific part of planning or operating electric buses. The utilized models and solution approaches are limited in their scope, for example regarding the consideration of heterogeneous fleets, TCO and infrastructure requirements. Charging infrastructure is mostly seen as an input to the problem, and charger optimization is thus not considered.

The present work addresses this gap and provides a highly customizable framework for the strategic planning of electric bus fleets. The framework is based on a grouping genetic algorithm (GGA), with incorporated mixed-integer charger optimization. The objective is to minimize TCO of the entire bus system. The presented GGA was inspired by Falkenauer [30], who emphasized the grouping aspect of scheduling problems. Its application to Pankratz's "Pickup and Delivery Problem" proved its ability to find high-quality solutions [31] for this class of vehicle routing problems. Furthermore, the work of Hosny and Mumford underline the superiority of GGA compared to other genetic algorithms for routing problems [32]. The GGA is advantageous due to the various options for evaluating a solution in the fitness function, which allows an effective integration of further constraints, parameters, and even sophisticated battery aging models. An application of the GGA to electric vehicle scheduling problems has not been reported in the literature so far.

The paper is structured as follows. Section 2 provides a general overview of the problem and introduces the formal problem formulation. Section 3 describes the solution approach with the developed "Grouping Genetic Algorithm" which includes a "Mixed Integer Non Linear Programming" (MILP) formulation. Subsequently, a case study based on the developed approach is presented in Section 4, and Section 5 finally summarizes the work and provides a conclusion.

\section{Problem definition}

The process of designing battery electric bus systems differs significantly from that of designing conventional diesel bus systems. In this section, first a general overview of the EVC-FMC is provided in Section 2.1. Next a mathematical formulation of the EVS-FMC is provided in Section 2.2. Although this mathematical formulation is intractable and cannot be solved directly with a general purpose solver even for small cases, its function is dual as providing a formal problem description, as well as being solved partially in the charger optimization presented in Section 3.2.2.

\subsection{General overview of the EVS-FMC}

Bus operators have optimized their diesel bus planning and procurement over decades. New buses are purchased on a regular basis to maintain an appropriate average age of the fleet. In the procurement process, decisions on bus types are mainly based on passenger capacity (length of the bus), comfort features such as the air conditioning system, and maximum power of the propulsion system. Driving range is not an issue due to the high energy density of diesel. However, the same is not the case for battery buses. The range of a battery bus is limited by the energy capacity of its battery. A larger battery increases the range, but also increases the weight of the bus and thereby raises the energy consumption per distance measure, thus increasing operational costs. Although several promising energy storage technologies are under development $[33,34]$, lithium-ion batteries with limited capacity are expected to be the predominant solution in the near future. Consequently, the fleet mix is an important part of the EVS-FMC.

The complexity is furthermore increased by the required charging time of electric buses, during which the buses are not available for service trip operation. The EVS-FMC focuses on depot charging battery buses, also known as "back-to-home" or "overnight charging", with charging infrastructure only installed at the depot. The majority of bus operators prefer this operating scheme, because the entire charging

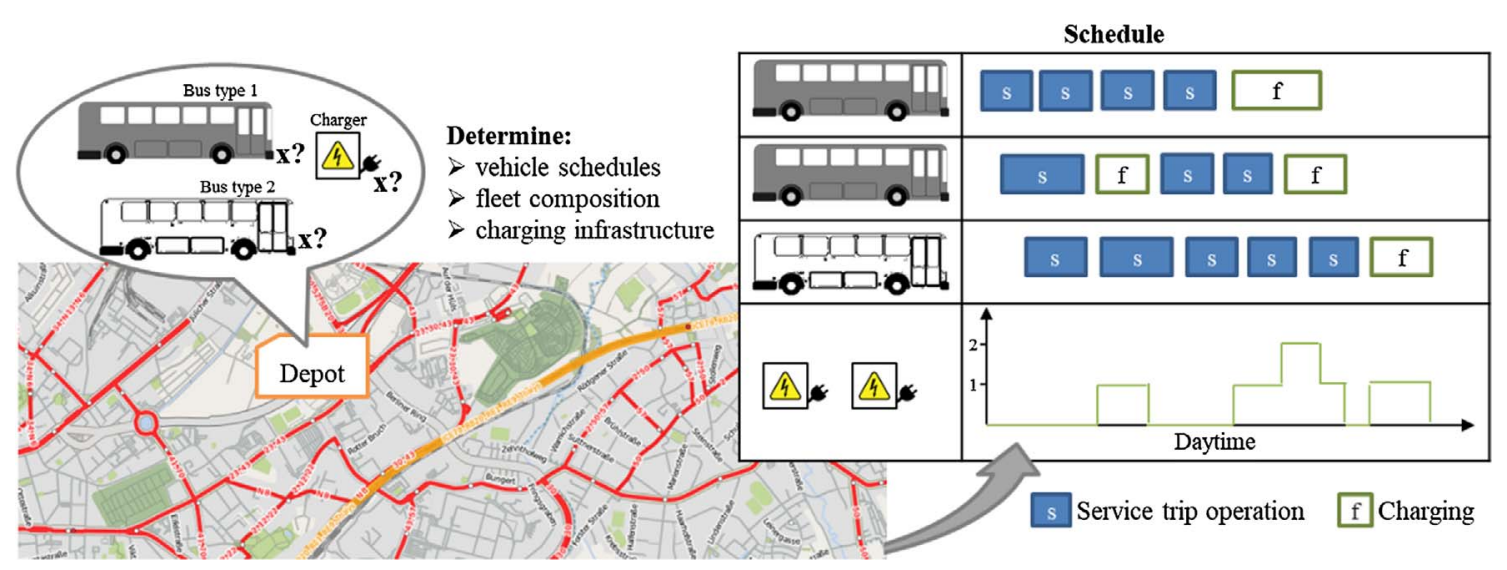

Fig. 1. Overview of the EVS-FMC. Outcomes are highlighted on the right by exemplary vehicle schedules and the corresponding number of required chargers. 
infrastructure can be installed on their own property. Furthermore, Daimler and MAN, two of the largest bus manufacturers in Europe, recently announced that their new electric product portfolio will focus on this technology.

An alternative measure to handle the range limitation of battery buses is to charge during operation. The so-called opportunity or fastcharging battery buses utilize high charging power levels to recharge within the existing dwell time at bus stops. This scheme aims to minimize the adjustments of the existing vehicle schedules and is excluded from the scope of this paper due to the different planning process that it requires.

The vehicle scheduling of depot charging battery buses has to consider bus-specific and trip-dependent energy consumptions, range limitations, and required charging times. The overall TCO of the system is determined by the vehicle schedule, as the required number of buses and chargers as well as the deadhead mileage and driver time follow directly from the schedule. Fig. 1 sketches the EVS-FMC that determines vehicle schedules, fleet composition, and charging infrastructure. Input consists of a given set of service trips (blue blocks in the right part of the figure), a set of battery bus types (e.g. "white" and "grey" buses), one charging station type, and a bus depot. Output, as depicted on the top right, assigns a sub set of trips to a bus of a specific type, together referred to as the vehicle schedule, and includes charging time. Furthermore, the number of chargers is determined as well, and the usage of these charges over time follows directly from the vehicle schedule.

The selection of bus types and quantities has to be done jointly with the scheduling of service trips to ensure that the particularities of each bus type are considered properly. Buses with a small battery capacity, for example, require several charging events during the daytime, whereas high-battery-capacity buses are charged mainly at night, which directly influences the fleet size and the number of required chargers.

Each service trip in the EVS-FMC is defined by a starting time and location, an ending time and location, and a set of route characteristics. The trip has to be operated by exactly one bus of the given bus type. Each bus type has a limited battery capacity, a specific energy consumption depending on the operational conditions, a fixed cost for the purchase of the bus, and energy-related costs for the battery usage, which represent the replacements costs for a degraded battery, or battery leasing. Each bus has to start and end its shift at the depot.

The total energy consumption in operation is limited to the allowed range for the state of charge of the battery, defined by $\mathrm{SOC}_{\min }$ and $S_{\text {SOC }}$. It is assumed that the state of charge equals SOC $_{\max }$ when the bus starts its shift, which implies a full charging during the night break. Charging takes place every time a bus visits a charging station, and it is done until the state of charge reaches SOC $_{\max }$ with constant power.

\subsection{Formal problem formulation}

For a set of $n$ service trips $S$, a set of possible charging events $F$, and a depot represented by a source node 0 and sink node $n+1$, the model of the EVS-FMC is defined on the directed multigraph $G=(N, A)$ depicted in Fig. 2. The set of nodes $N$ is defined as $N:=S \cup F \cup 0_{s} \cup 0_{f} \cup q_{s} \cup q_{f}$. Thus, the set $\mathrm{N}$ contains a node for each service trip $i \in S$ represented by blue nodes in Fig. 2; it contains a node for each possible start of a charging event $f \in F$ represented white nodes in Fig. 2; and it also contains source nodes $0_{s}, 0_{f}$ and sink nodes $q_{s}, q_{f}$ representing the depot for both the set of service trips and the set of charging events, respectively. The set of $\operatorname{arcs} A, A:=A^{s} \cup A^{f}$ consists of the set of all feasible deadhead trips $A^{s}$, represented by solid arcs in Fig. 2, and the set of arcs $A^{f}$ that connects charging events serviced by the same charger, represented by dashed arcs in Fig. 2. A deadhead trip represents a non-service trip where a bus drives from either the end terminal of one service trip to the start terminal of the next service trip, from the end terminal of a service trip to a charger, or from a charger to the start terminal of a service trip. Furthermore, $S_{0}$ denotes the union of the set of service nodes $S$ and the source node $0_{s}, S_{n+1}$ denotes the union of the set of service nodes $S$ and the sink node $q_{s}$, and $S_{0, n+1}$ denotes the union of the set of service nodes $N^{s}$, the source node $0_{s}$ and the sink node $q_{s}$. Similarly, the set $F_{0}\left(F_{n+1}\right)$ denotes the union of $F$ and the source node $0_{f}$ (sink node $q_{f}$ ). The solution to the EVS-FMC defines a set of paths in the graph $G$ depicted in Fig. 2 such that all (blue) service nodes are contained in exactly one path. Charging events are inserted in the paths to ensure sufficient charge for the full trip, and a fully charged battery of each bus by the end of the day.

A service trip $i \in S$ is defined by a start time $\beta_{i}$, a duration $t_{i}$, and a set of trip characteristics that determine the energy consumption, such as the geographical route, number of stops, and height profile of the route.

Let $V$ be the set of bus types. Each bus type $k \in V$ is represented by a usable battery capacity $E^{k}$ in the range from $S_{\text {max }}$ to $S_{\text {min }}$, a recharging capability $r^{k}$, and a specific energy consumption of $h_{i j}^{k}$ representing the energy costs of servicing trip $j \in S_{n+1}$ after trip $i \in S_{0}$ using vehicle type $k \in V$, including costs of servicing trip $i$ and deadheading from $i$ to $j$. Let $m^{k}$ be the purchasing cost of a bus of type $k \in V$, and $c_{e}^{k *}$ the bus-specific energy costs. The time-related operational costs of a bus (mainly driver salary) are represented by $c_{t}$. The time required for the subsequent execution of event $j \in S_{n+1}$ after $i \in S_{0}$ is expressed by $t_{i j}$. A supplement of $15 \mathrm{~min}$ is added to all trips coming from and going to the depot to consider the required handling for parking and connecting to the charging infrastructure. The charging time is not considered in the operational costs as in-service time costs, because the charging is not monitored by the driver. Purchasing costs of charging infrastructure are defined per charger as $p$.

Let the binary decision variable $x_{i j}^{k}$ equal 1 , if a bus of type $k \in V$ is operated on arc $a \in A^{s}$, which means that service trips or charging events are performed subsequently with the same bus type. Let the binary decision variable $z_{l m}$ equal 1 , if charging event $m \in F$ takes place after charging event $l \in F$; thus, the arc $(l, m) \in A^{f}$ connects the charging events. The current energy level of a bus after executing trip $i \in S_{0, n+1}$ is defined by $e_{i}^{k}$. Let $a_{i}$ be the starting time of event $i \in S \cup F$ (service trip or charging event) and $a_{i}=\beta_{i}$ for all $i \in S$. The starting time $a_{i}$ for charging events $i \in F$ must be in the range of the first

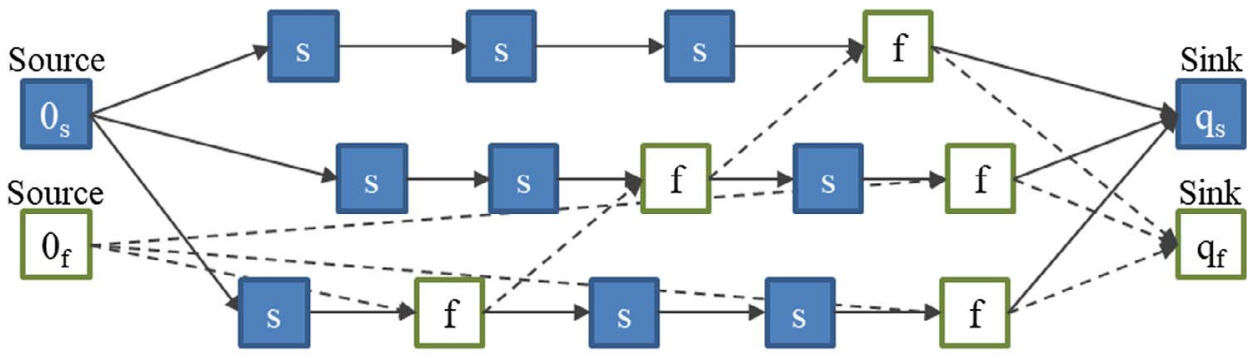

Fig. 2. Directed multigraph of the EVS-FMC with arcs $A^{s}$ connecting service trips and charging events operated by the same vehicle, and arcs $A^{f}$ connecting charging events operated by the same charger. 
possible charging time $\gamma$ and the last possible charging time $\delta$. The constant $M \gg a_{i}$ is used to avoid nonlinearities.

\section{Objective function}

$$
\begin{aligned}
& \min \sum_{k \in V} \sum_{j \in N} m^{k} x_{0 j}^{k}+\sum_{k \in V} \sum_{i \in S_{0}, j \in N, i \neq j}\left(t_{i}+t_{i j}\right) c_{t} x_{i j}^{k} \\
& +\sum_{k \in V} \sum_{i \in N, j \in F, i \neq j} c_{e}^{k *}\left(E^{k}-e_{j}^{k}\right) x_{i j}^{k}+\sum_{j \in N} p z_{0 j}
\end{aligned}
$$

The objective (1) is to minimize the TCO, which is split up into four sums. In the first sum, the bus investment is calculated based on the arcs leaving the depot from the source node. Secondly, in-service costs unrelated to energy consumption, such as bus driver costs, are accumulated and multiplied with a time-dependent cost factor. The third term represents the bus-specific costs related to the energy consumption. It considers the accumulated energy consumption whenever a bus visits a charging station, multiplied by an energy-dependent and bus-specific cost factor. Finally, the last part of the sum represents the investments in charging infrastructure.

\section{Vehicle scheduling constraints}

$\sum_{k \in V} \sum_{j \in S_{n+1}, i \neq j} x_{i j}^{k}=1 \quad \forall i \in S$

$\sum_{k \in V} \sum_{j \in S_{n+1}, i \neq j} x_{l j}^{k} \leqslant 1 \quad \forall l \in F$

$\sum_{i \in S_{n+1}, i \neq j} x_{j i}^{k}-\sum_{i \in S_{0}, i \neq j} x_{i j}^{k}=0 \forall j \in N, \quad \forall k \in V$

$a_{i}+t_{i}+t_{i j} \leqslant a_{j}+M\left(1-\sum_{k \in V} x_{i j}^{k}\right) \quad \forall k \in V, \forall i \in S, \forall(i, j) \in A$

$x_{i j}^{k} \in\{0,1\} \quad \forall k \in V, i \in S_{0}, j \in S_{n+1}, i \neq j$

Constraint (2) ensures that each service trip is covered by exactly one bus, while constraint (3) enforces that each copy of a charging station can maximally be visited once. Constraint (4) represents the flow constraints of the vehicle network. Next, constraint (5) ensures that events assigned to one vehicle do not overlap in time - that is, the starting time of the next service trip or charging event for a vehicle is greater or equal to the sum of the starting time of the previous trip, the trip or charging duration, and the deadheading time. In vehicle scheduling, the start time of service trips is pre-defined by the timetable, while the start time of charging events is determined in the model. Constraint (6) defines the binary nature of the decision variables $x_{i j}^{k}$, representing that a vehicle of type $\mathrm{k}$ visits event $\mathrm{i}$ after event $\mathrm{j}$. Events could be both service trips and charging events. Individual vehicle schedules are obtained from the $x_{i j}^{k}$ variables of a solution.

\section{Energy consumption constraints}

$0 \leqslant e_{j}^{k} \leqslant\left(e_{i}^{k}-h_{i j}^{k}\right) x_{i j}^{k}+E^{k}\left(1-x_{i j}^{k}\right) \quad \forall k \in V, \forall i \in S, \forall j \in S_{n+1}, i \neq j$
$e_{0}^{k}=E^{k} \quad \forall k \in V$

$0 \leqslant e_{j}^{k} \leqslant E^{k}-h_{l j}^{k} x_{l j}^{k} \quad \forall k \in V, \forall l \in F, \forall j \in S_{n+1}, i \neq j$

Constraint (7) ensures that the required energy level for the assigned trips to a vehicle never exceeds the usable battery capacity. Furthermore, constraints (8) and (9) ensure that buses are fully charged when leaving the depot and after charging, respectively - that is, that the battery energy level $e_{0}^{k}$ is set to the maximum.

Charger scheduling constraints

$\sum_{l, \in F_{n+1}, l \neq m} z_{m l}-\sum_{l \in F_{0}, l \neq m} z_{l m}=0 \quad \forall m \in F$

$\sum_{m \in F_{0}} z_{l m}=\sum_{k \in V} \sum_{i \in S} x_{i l}^{k} \quad \forall l \in F$

$\sum_{k \in V} \sum_{i \in S} x_{i l}^{k}\left(a_{i}+t_{i}+t_{i l}+r^{k}\left(E^{k}-e_{i}^{k}\right)\right) \leqslant \sum_{k \in V} \sum_{j \in S} x_{j m}^{k}\left(a_{j}+t_{j}+t_{j m}\right)$

$$
+M\left(1-z_{l m}\right) \quad \forall l, m \in F
$$

$\gamma \leqslant a_{m} \leqslant \delta \quad \forall m \in F$

$a_{l}+r^{k}\left(E^{k}-e_{l}^{k}\right)+t_{l j} \leqslant a_{j}+M\left(1-\sum_{k \in V} x_{l j}^{k}\right) \quad \forall k V, \forall l \in F, \forall(l, j) \in A$

$z_{l m} \in\{0,1\} \quad \forall l, m \in F, l \neq m$

Constraint (10) represents the flow constraints of the charging infrastructure network, while constraint (11) ensures that each charging event has a corresponding ingoing trip. Next, constraint (12) defines that the starting time of the next charging event has to be greater or equal to the ending time of the previous charging event. Constraint (13) enforces that the charging can only be done within the pre-defined time window. Moreover, constraint (14) ensures that no charging events for a single charger overlap in time. Finally, constraint (15) defines the range of the binary decision variable $z_{l m}$ indicating whether two charging events are scheduled sequentially.

\section{Solution approach}

The developed approach aims to provide a straightforward toolchain for the strategic planning of electric public transport bus fleets. It is based on the input data of bus operators, which is enriched by additional information and finally utilized within a genetic algorithm that evaluates alternatives regarding fleet composition and scheduling. The overall framework is illustrated in Fig. 3.

The input data (A) consists of operational and technical information. It is utilized in the preprocessing (B) to calculate the values, which are not influenced by the vehicle scheduling, as for example the deadhead mileage between terminal stops. The route determination ensures that all distances of potential deadhead trips between service trips and between a service trip and charging station at the depot are realistic. The data prepared in the preprocessing is used within the GGA (C), which generates a population in which each individual describes a

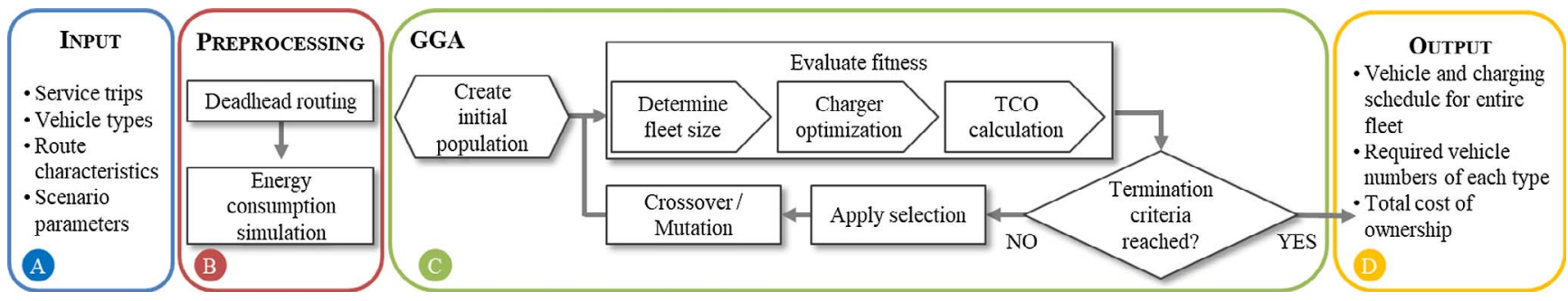

Fig. 3. Framework of the solution approach. 
feasible assignment of an entire service trip set to of sub schedules (blocks) starting and ending at the depot. In the following charger optimization phase, vehicle schedules including charging times are designed based on these blocks. Individuals in the population are evaluated regarding their TCO, consisting of electric bus procurement costs (including determination of fleet size and mix), charging infrastructure investments, and operational costs consisting of energy and personnel costs. After reaching a termination criterion, the GGA delivers the required fleet size and mix, the corresponding schedules, and the TCO of ownership as output (D). The individual steps are described in further detail in the following.

\subsection{Preprocessing}

A focus of this work is the ability to handle real-world scenarios with limited manual effort. Starting from the data export of current operational planning software, the set $S$ of service trips and the set $F$ of possible charging events are generated. Each service trip $s \in S$ described by starting time $\beta_{i}$, duration $t_{i}$, passenger load, elevation profile, and start and end location. The location of the depot is also provided. Each vehicle type $k \in V$ is described by the empty vehicle weight, the energy density of the battery system, the efficiencies of the traction system, and the air drag and rolling resistance parameters. The scenario parameters cover cost figures and lifetimes of vehicles and charging infrastructure, as well as a value describing the planning horizon.

Based on this input, the deadhead routing and the simulation of energy consumption for all service and deadhead trips are performed in an automatized preprocessing. The Graphhopper routing service (www. graphhopper.com) is utilized to gather road distances between a matrix of geo locations that are described here by the start and end points of service trips and the location of the depot. The distances are subsequently utilized to calculate duration $t_{i j}$ and energy consumption per deadhead. The bus routes are modeled in more detail directly in the geo database Openstreetmap (www.openstreetmap.org) to account for bus stops and corresponding dwell times.

The energy consumption depends on the bus type as well as on several trip characteristics: the length of the route, the average speed, the expected passenger load per trip, and the slope of the route. Therefore, the energy consumption is computed in a simulation. Sinhuber describes the applied energy consumption simulation methodology, and it has already been utilized in previous work $[35,36]$. A model is configured for each bus type, so that varying weights and efficiencies are taken into account. Thus, the energy consumption values $h_{i j}^{k}$ of servicing trip $i$ and deadheading from trip $i$ to trip $j$ are computed for every individual bus type, and serve as input to the algorithm. Varying energy consumptions, for instance caused by weight differences between vehicle types, can be taken into account, and their influence on the TCO can be evaluated.

\subsection{Grouping genetic algorithm}

The heuristic is based on Falkenauer's concept describing the GGA [30]. It emphasizes the grouping aspect of a scheduling problem - in this case, the assignment of a set of service trips to blocks. This clustering is the core part of the EVS-FMC problem, as the locations and times of service trips are already fixed and the assignment of a feasible set of service trips $s \in S$ to blocks uniquely defines the tour. The GGA has been chosen because its ability to find high-quality solutions for comparable problems [31]. Furthermore, it is well suited for parallel computing and offers several options for problem-specific customization.

The general procedure of the GGA is shown in step C of Fig. 3. It is started by the generation of an initial population (set of individuals) that is afterwards evaluated by the fitness function. The process of evaluating an individual consists of three steps: (1) the determination of the fleet size by combining trip sequences defined by the chromosomes to entire day vehicle schedules, (2) the charger optimization, and (3) the TCO calculation. If no termination criterion is reached, the process continues with the selection of competitive individuals, which are modified in the crossover and mutation operation. After new genetic material has been created by crossover and mutation, the entire generation is evaluated again and the loop continues until a termination criterion is reached. Termination criteria can, for example, be formulated as a maximum number of iterations, a limit for the change of the best fitness value over the last iterations, and a lower bound of the diversity. The implementation in this work is terminated after a maximum number of iterations, but the extension to any of the other measures is straightforward.

The described procedure applies to the majority of genetic algorithms. The particularity of the GGA is its genetic representation of individuals. The genes of the GGA represent the characteristics of groups, which are in our case blocks of service trips. The variable length of blocks implies therefore chromosomes of a variable length. Furthermore, special operators are needed to perform crossover and mutation on the level of blocks.

\subsubsection{Genetic representation and operators}

The clustering of service trips within the GGA is done by the creation of blocks that also define the genetic representation (chromosomes), as shown in Fig. 4. A block represents a trip sequence operated by the same vehicle, starting from and ending at the depot. Each block is followed by a charging event, and each individual in the population of the GGA covers the entire set of service trips divided into these blocks.

The block-based representation differs from standard ones, such as binary string chromosomes or arrays with real-valued numbers, as it is especially suited for clustering problems. The chromosome consists of different blocks to which service trips (represented as blue dots in Fig. 4) can be assigned. The order of service trips within a block is uniquely defined by the starting time of the service trip. Each block is assigned to one bus type so that the specific usable battery capacity given the bus type's specific energy consumption is respected. The blocks are furthermore characterized by a unique identifier (ID) that represents a consecutive numbering according to the earliest starting time of the covered trips.

The number of blocks in an individual and the number of trips assigned to a block can vary. Furthermore, the total number of required buses is not defined at this stage: it is computed afterwards in the fitness function by combining blocks and charging events to construct full-day vehicle schedules. This is done jointly with the charger optimization, as described in section 3.3. The GGA only allows the construction of feasible blocks, in which the energy required to service all trips does not exceed the battery capacity and there exists a feasible ordering of the assigned trips in time. An ordering is feasible when the start time of the next trip is no earlier than the time required to service the previous trip and deadhead to the following one. The subsequently described construction heuristic ensures that only feasible blocks in terms of time and energy exist in the population.

The population is divided into several subpopulations that are computed in parallel. After a number of generations, a migration is performed in which individuals are exchanged among the subpopulations. The exchange strategy is to replace the worst individuals of one subpopulation with the best individuals of another. The separated treatment allows for promising solutions to be developed independently

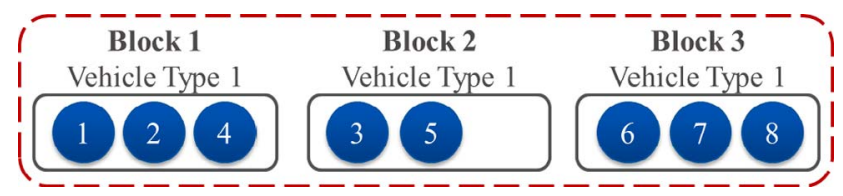

Fig. 4. Genetic problem representation. 


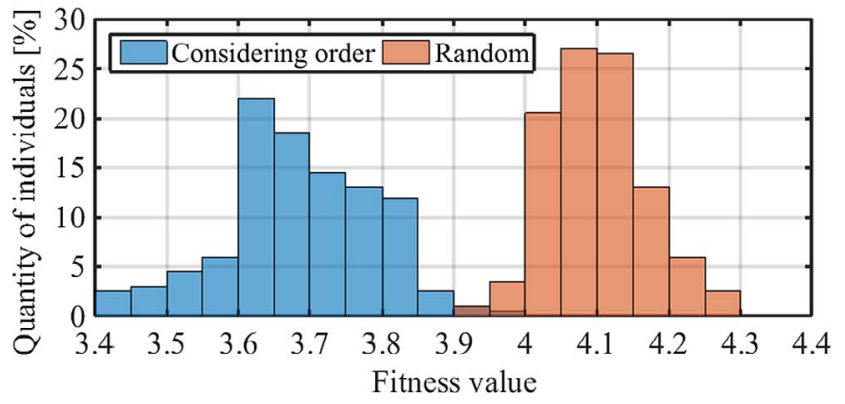

Fig. 5. Distribution of fitness values ( $€$ per service $\mathrm{km}$ ) of the initial solutions for greedy and random start.

for a certain time, and the experiments conducted in this study reveal an improved stability of the algorithm.

Initial solution. Both a random and a greedy initialization are implemented and evaluated. In the random initialization, a trip is selected at random and inserted according to the insertion heuristic described in the next paragraph. In the greedy initialization, in contrast, trips are inserted based on their starting time. Given a randomly selected first trip and a randomly selected direction (forward or backward in time), the trips to be inserted are ordered according to starting time and selected in this order. Although the first random initialization leads to a higher diversity, the quality of the initial solutions is generally worse as they may contain large gaps in time. Conversely, the diversity of the greedy initialization is lower, but solutions are generally of higher quality as the blocks are denser.

Fig. 5 provides an exemplary overview of the quality of the initial solutions of both approaches. The experiments have shown that the greedy start enables the GGA to determine better overall solutions. Thus, this methodology is set as the standard for this work.

Insertion heuristic. The insertion heuristic, which is included in the crossover and mutation operators, assigns service trips to blocks based on operational costs. The service trip that should be inserted is selected beforehand by the calling function of the insertion heuristic. The heuristic inserts a service trip into a block for which the additional operational costs are the lowest.

For a given service trip, the insertion costs are calculated for all existing blocks as well as for creating a new block. The costs consist of the energy-related expenditures (electricity and battery usage) and the operational cost of deadheading to and from the service trip. Two scenarios could arise in which an insertion is impossible. First, the timing could be infeasible, meaning that the existing and the given service trip cannot be combined. Second, the required energy level could be insufficient. The new block considered by the insertion heuristic is empty and, in case of an assignment, would cover only the given service trip with its pull-out and pull-in deadhead trips and a time buffer for handling at the bus depot. The consideration of an empty block ensures the existence of a feasible assignment.

Crossover. The crossover operator generates new genetic material by combining the chromosomes of selected parents. Blocks of the first parent are combined with blocks of the second parent to form an offspring. Fig. 6 illustrates the crossover process.

First crossover points indicated by yellow vertical lines in Fig. 6-I are defined for both parents: two for the donating parent and one for the receiving parent. In the second step, the blocks between the two crossover points of parent 1 are copied to parent 2 at the position of the defined crossover point. The resulting child in stage II is infeasible. The insertion from parent 1 has led to the issue of trips occurring twice in the child. Steps III to VI repair the solution. In step III, blocks of parent 2 with an ID equal to the inserted blocks of parent 1 are removed. Step IV removes remaining duplicate service trips from parent 2 , i.e. trips that are contained in the inserted blocks of parent 1 . Then, in step V, any service trips that are not contained in the child (as a result of step III) are re-inserted in random order according to the insertion heuristic. The result in step VI is the new feasible offspring produced from the genetic material of both parents.

Mutation. The mutation aims to generate new genetic material to increase diversity. Instead of combining chromosomes of individuals, as in the crossover, it modifies only single individuals. The mutation deletes one block selected at random, and then reinserts the deleted service trips in random order. The feasibility of the solution is ensured by the usage of the insertion heuristic.

Fitness function and selection. In the construction of blocks, only operational costs are considered. The fitness function evaluates the solution with regard to the TCO, consisting of operational costs, number

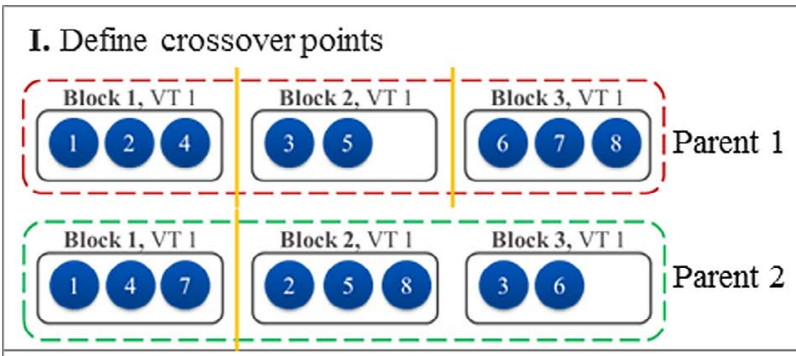

II. Copy chromosomes and generate offspring

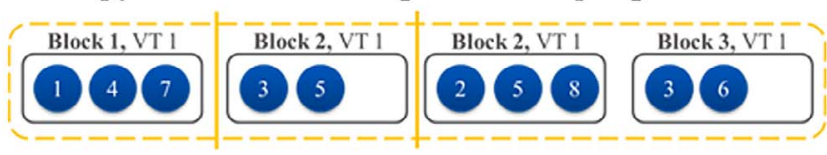

III. Delete blocks with identical ID, keep copied elements

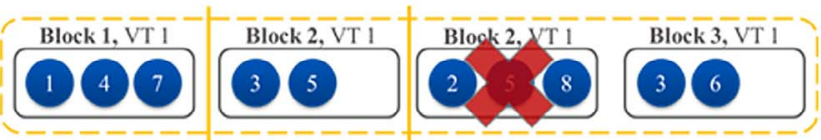

IV. Delete duplicated trips

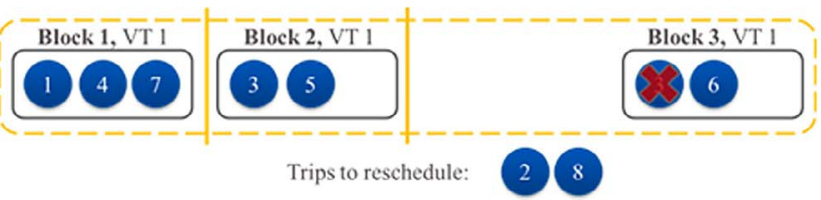

V. Reschedule trips with insertion heuristic and update IDs

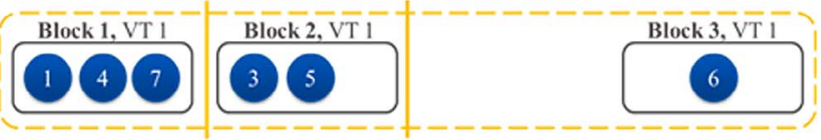

Trips to reschedule:

VI. Result

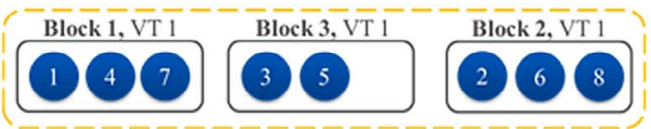

Fig. 6. Overview of the implemented crossover process. 
of required buses, and number of required chargers.

The fitness function is divided into three steps, as shown in part $\mathrm{C}$ of Fig. 3. First, the fleet size is determined. This comprises the calculation of charging time required after completion of one block and the assignment of blocks to buses. It is assumed that each bus can start charging immediately after arriving at the depot. The assignment is done based on a "first charged first out" strategy, which means that the first bus to be fully recharged serves the next block. This defines the fleet size and composition.

However, when charging takes place immediately after a bus arrives at the depot, several simultaneous charging events can occur. This happens especially in the evening when the passenger service is gradually reduced and the buses return to the depot. Therefore, charging events are rescheduled in the second step of the fitness function to reduce the number of chargers and the overall power level at the bus depot. Section 3.2.2 describes how the rescheduling is implemented and discusses further strategies for the integration in the overall framework.

Finally, the TCO is calculated for each individual in step 3. It sums up the investment depreciations and the operational expenses of a given time period. Within the GGA, the TCO characterizes the quality of an individual and is therefore called fitness value.

In the selection process, individuals are ranked based on their fitness value. A stochastic uniform selection process is used to select individuals for reproduction. They form the input for the next generation and are modified by crossover or mutation. The selection process can be described using the example of a line on which each parent has a share according to the square root of its rank. A selector proceeds along the line in equal step sizes and selects parents according to the sections, starting with a random point smaller than the step size. This concept can also be described as a roulette wheel with several pointers in an even distance.

\subsubsection{Optimization of charging infrastructure}

The objective of the charger optimization is to reduce the number of chargers and therefore the charger investments. The input is described by a set of charging events $F$, which have an earliest possible starting time $b_{l}$ and a duration $d_{l}$. The charger optimization assigns charging events to chargers. The rescheduling of the charging events to reduce the number of chargers and the grid load is a complex scheduling problem in itself. However, the number of charging events in $F$ is significantly lower than the number of service trips described by the set $S$. Thus, common MILP solution approaches can be utilized. The mathematical model is derived from the network flow problem formulation presented in Section 2.2 and uses the already introduced notation. It is parameterized during runtime in Matlab [37] and solved by CPLEX [38]. Penalties for shifting a charging event in time are included, as this has been found to decrease computation time. However, the weighting factor $w$ is defined to ensure that a reduction in the number of chargers always dominates potential shift penalties.

\section{Without rescheduling of charging events \\ I $\rightarrow$ Assignments could appear worse than they are}

SCHEDULE BEFORE MUTATION / CROSSOVER

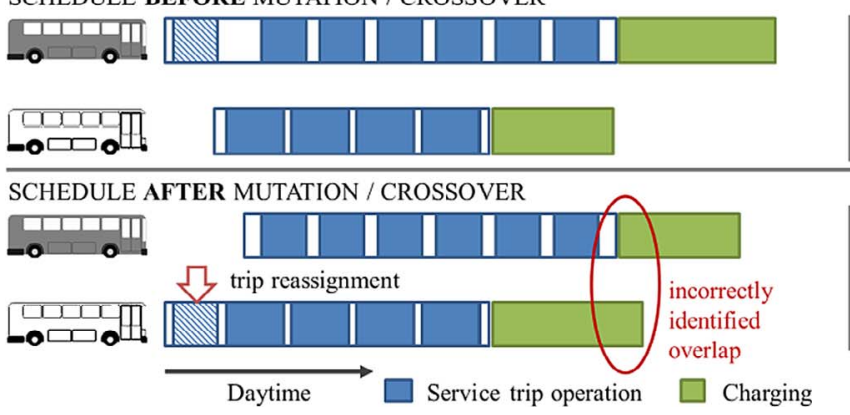

$$
\min \sum_{m \in F} z_{0 m}+w \sum_{l \in F} u_{l}
$$

s. t. $\sum_{l \in F_{n+1}, l \neq m} z_{m l}-\sum_{l \in F_{0}, l \neq m} z_{l m}=0 \quad \forall m \in F$

$\sum_{l \in F} z_{l m}=1 \quad \forall m \in F$

$a_{l}+d_{l} \leqslant a_{m}+M\left(1-z_{l m}\right) \quad \forall m, l \in F$

$\gamma_{l} \leqslant a_{l}+d_{l} \leqslant \delta_{l} \quad \forall l \in F$

$a_{l}-b_{l} \leqslant u_{l} \quad \forall l \in F$

$u_{l} \in \mathbb{R}_{+} \quad \forall l \in F$

$z_{l m} \in\{0,1\} \quad \forall l \in F, m \in F, l \neq m$

The objective in (16) minimizes the number of chargers (first term), as well as the amount of time-shift (second term). Constraint (17) is the flow conservation constraint, while constraint (18) enforces that every charging event is scheduled. The linkage of charging events and therefore the usage of the same physical charger is handled by constraint (19), which ensures that the subsequent charging event can only be linked if the starting time $a_{j}$ is greater than the ending time of the previous event. Furthermore, constraint (20) limits the overall time window of charging events. Finally, the shift penalties are defined by constraint (21) as a supplement $u_{i}$ added to the starting time, and added for faster convergence.

Several measures are introduced to accelerate the computation of the model so that an integration into the fitness function is possible. The number of connection arcs is reduced by using ordered sets and only allowing forward assignments $z_{l m}=0 \forall l \in F, m \in F, l \geqslant m$. The weighting factors are configured in such a way that the charger costs always dominate the shift penalties. A limit on the optimality gap is used to stop the computation if the number of chargers cannot be reduced further. In addition, a time limit of $5 \mathrm{~s}$ is introduced, which stops the solving and uses the best solution found at that point, even if optimality is not proven. Instances with up to 70 charging events are solved in less than $1 \mathrm{~s}$ with CPLEX.

Including the MILP formulation in the fitness function enables the genetic algorithm to evaluate and select individuals based on the TCO, which includes the costs of charging infrastructure. Alternative strategies would be to approximate or ignore the number of chargers in the fitness function and to conduct the charger optimization afterwards.

An approximation could be realized by disabling the rescheduling of charging events in the fitness function, as shown by strategy I, illustrated in the left part of Fig. 7. This would lead to every charging event starting immediately after a bus has finished a block and arrives at the depot. In the example in Fig. 7, both charging events can be operated by one charger. However, due to the reassignment of the initial trip of the

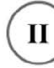

Not considering charger costs at all

$\rightarrow$ Assignments could appear better than they are

SCHEDULE BEFORE MUTATION / CROSSOVER

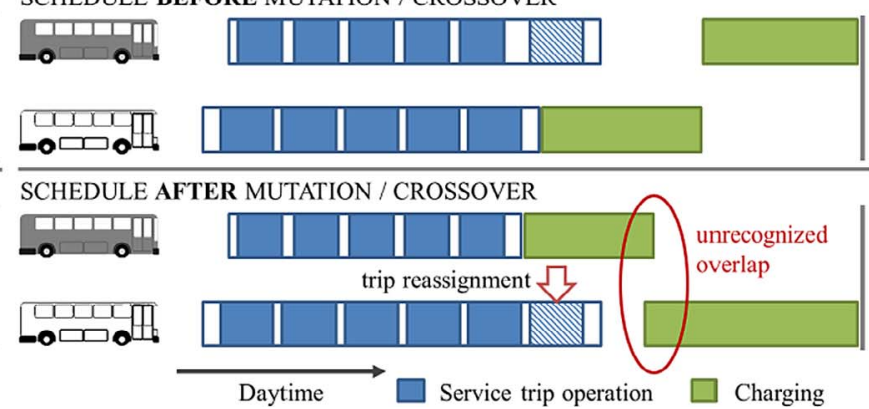

Fig. 7. Illustration of problem cases that occur when using approximation strategies for determining the number of chargers. 
first bus during mutation or crossover, the charging time of the second bus increases. The consequence is an overlap and the conclusion that two chargers are required. Thus, the number of chargers is overestimated, because the potential shift forward in time is not utilized. The problem also occurs for improvements in the scheduling of service trips that would enable a reduction in the number of chargers. The improvements could be hidden and therefore not considered by the genetic algorithm, if the number of chargers is approximated with an overestimation.

Not considering charging investments at all during the GGA runtime would lead to the opposite problem (strategy II, right part of Fig. 7). Unfavorable assignments of service trips that would cause an increased number of chargers would appear better than they are and could be misleading for the genetic algorithm. The example highlights how the reassignment of the last trip of the first bus during mutation or crossover leads to an increased charging time of the second bus and a shift forward in time of the entire charging event, because the bus arrives later at the depot. Depending on the reduction of the charging time of the first bus, which is influenced by the charging power capabilities of the bus type and the difference in energy consumption before and after the reassignment, it is possible for the two charging events to overlap. By using the approximation, this overlap would not be visible for the GGA. A subsequent charging optimization could not fix this issue.

\section{Case study}

The developed methodology provides a straightforward process to define a fleet of depot charging battery buses and their corresponding charging infrastructure for real-world problems. In this case study, the methodology is applied to two scenarios that differ in their mode of operation. The following introduces the particularities of both scenarios, and subsequently discusses the computed results.

Comparable case studies have been initiated in several cities worldwide by bus operators and transport authorities. Their aim is to assess the technical feasibility and the financial consequences of an electrification. However, the majority of these studies are limited to a purely technical evaluation $[29,39]$, without taking advantage of vehicle schedule adjustments. The common methodology is to take the existing vehicle schedule of diesel buses, calculate the required energy to fulfill the schedule, and compare this to the installed battery capacity of the electric buses. These studies do not consider modifications of the vehicle schedules, as proposed in this work, thereby limiting their scope.

\subsection{Scenario definition}

The scenarios analyzed in the following represent two different cities and modes of operation. Scenario A, in the German city of Aachen, represents a constant-frequency operation in an urban environment; and scenario B, in the Danish city of Roskilde, concerns an operation with different frequencies in the peak hours and operation on a more regional environment, in that distances are generally larger and the average speed of operation is slightly higher compared to the Aachen scenario. The scenarios thereby cover two typical modes of operation encountered by bus operators. In terms of fleet size, ranging from 12 to 14 diesel buses, the scenarios' scope corresponds to the yearly vehicle replacement of a mid-size bus operator.

The solution quality is evaluated and discussed in the following based on the fitness value. A comparison of the two scenarios is enabled by using the ratio of the total costs (investment and operational costs during the entire planning horizon) and the performed service mileage. The following section describes the route and operational characteristics of the scenarios in detail.

\subsubsection{Route characteristics}

Located in Aachen, scenario A consists of the bus routes 33, 73, and 173. Routes 73 and 173 are shortened variants of route 33 visiting other terminal stops (end point of a route). Fig. 8 shows the track of route 33 in its entirety, with a distance of about $11 \mathrm{~km}$. The vehicle schedules provided by the bus operator contain further trips on additional routes in the early morning and late evening. They are also modeled and considered in the following. In total, 23 differently located terminal stops are visited on the considered type of day.

Scenario B is located in Denmark and connects the city of Roskilde with the Copenhagen area. The length of the considered route, route 123 , is $23 \mathrm{~km}$ and therefore over twice as long as the route in scenario A. Furthermore, the mileage to the depot is higher, especially for the terminal stop in the east, as shown in Fig.8. In contrast to scenario A, only four different terminal stops of the bus route are visited on the considered type of day, which reveals a more homogeneous operation. Route 123 is highlighted in the right part of Fig. 8.

\subsubsection{Operational characteristics}

Scenario A represents a constant operation with a slight peak in the evening, whereas scenario $B$ refers to an operating mode with increased frequency in peak hours. Fig. 9 shows the simultaneous service trips and the number of diesel buses currently operated. The two peaks in the morning and afternoon are clearly visible for scenario B: the conventional diesel buses return to the depot in the off-peak hours. This natural pause in the depot seems to be favorable for the charging of battery buses.

Performance figures of the current diesel bus system are given in Table 1 . The average speeds reveal that scenario A has a dominating urban character, whereas scenario B reflects suburban operation. The reported runtime represents the accumulated time across all buses away from the depot and a handling offset of $15 \mathrm{~min}$ for every incoming or outgoing trip.

Further parameters assumed for the case study are provided in Table 2. They are utilized for both scenarios, assuming identical conditions for Germany and Denmark. Time-dependent costs are determined by the driver salary, with the assumed value corresponding to the German salary level. Energy-related costs consist of expenses for electricity consumption. The electricity price is a parameter of the scenario, whereas battery costs are defined individually for every bus
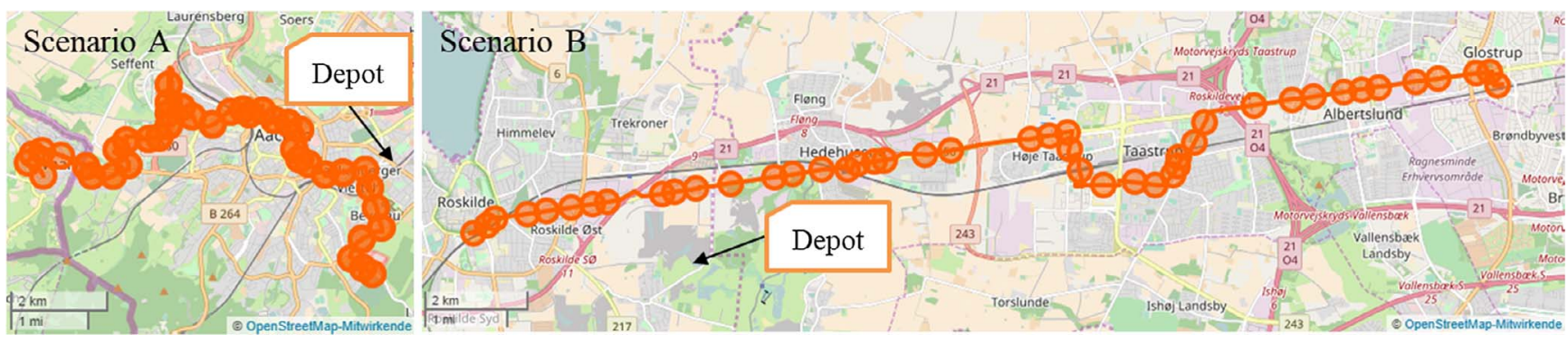

Fig. 8. Map of the main routes operated in scenarios A and B, and location of the bus depots. 

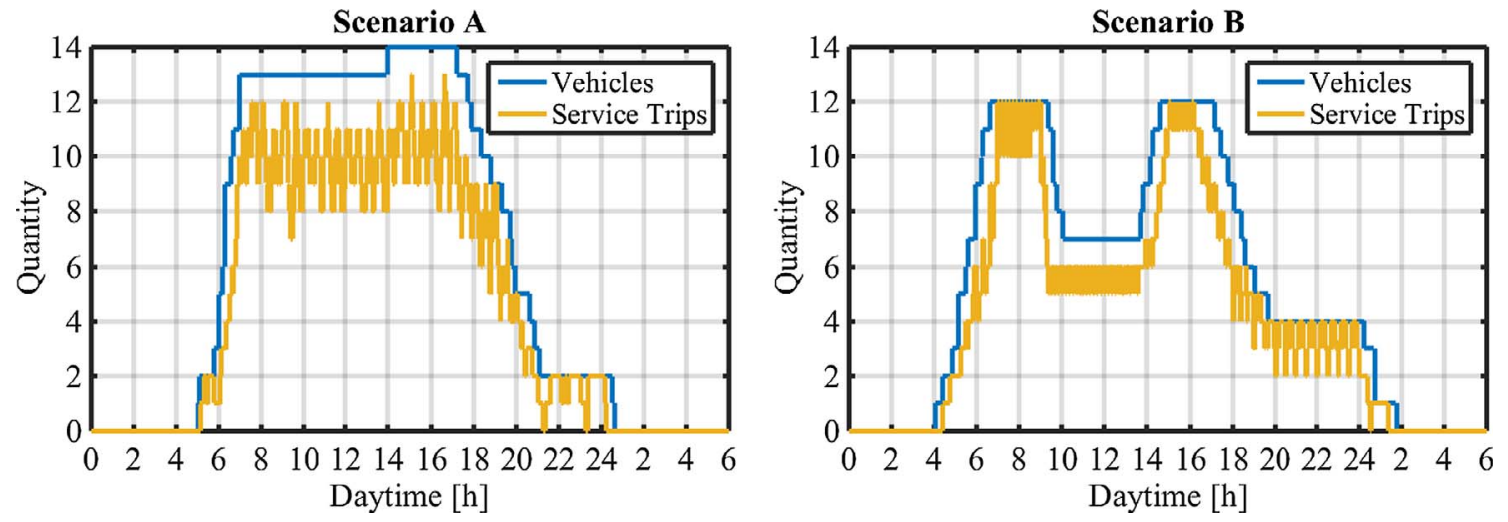

Fig. 9. Number of diesel buses and simultaneous service trips of considered scenarios with the original vehicle schedules.

Table 1

Operational figures of the conventional diesel bus system.

\begin{tabular}{lll}
\hline Parameter & Scenario A & Scenario B \\
\hline Day type & Weekday & Weekday \\
Number of service trips per day & 200 & 135 \\
Total service mileage per day $[\mathrm{km}]$ & 2198 & 3047 \\
Average speed $[\mathrm{km} / \mathrm{h}]$ & 16.3 & 24.6 \\
Average trip distance $[\mathrm{km}]$ & 11.0 & 22.6 \\
Max. buses in service per day & 14 & 12 \\
Deadhead mileage per day $[\mathrm{km}]$ & 254 & 363 \\
Bus runtime per day $[\mathrm{h}]$ & 189 & 160 \\
\hline
\end{tabular}

Table 2

Further case study parameters.

\begin{tabular}{ll}
\hline Parameter & Value \\
\hline Time-dependent costs [€/h] & 25 \\
Electricity price [€/kWh] & 0.25 \\
Investment costs charger [€/unit] & 60,000 \\
Planning horizon of bus and charger depreciation [years] & 12 \\
Average weight per passenger [kg] & 75 \\
Time at which every charging event has to be finished & 6 a.m. \\
\hline
\end{tabular}

type. Investment costs for charging infrastructure are defined as a fixed value for each charger. The planning horizon is required for the calculation of depreciations. It is assumed for the case study that the defined scenarios are operated on every day of the year. The average weight per passenger is essential for the energy consumption simulation, and the time limit defines the latest possible end of a charging event.

Table 3 summarizes the characteristics of the two bus types included in the case study. The technical parameters are based on the specifications of 12-meter battery electric buses from Optare and BYD. Both bus types offer the same transport capacity of about 4 tons, but their

Table 3

Considered battery bus types.

\begin{tabular}{lll}
\hline Parameter & Bus type 1 & Bus type 2 \\
\hline Total battery capacity $[\mathrm{kWh}]$ & 90 & 380 \\
Usable battery capacity $E_{\text {usable }}[\mathrm{kWh}]$ & 60 & 282 \\
Empty vehicle weight $[\mathrm{kg}]$ & 8000 & 14,000 \\
Gross vehicle weight $[\mathrm{kg}]$ & 12,000 & 18,000 \\
Investment costs bus w/o battery [€] & 250,000 & 350,000 \\
Bus depreciation period [years] & 12 & 12 \\
Investment costs battery [€/kWh] & 700 & 600 \\
Battery lifetime full cycle equivalents [FCE] & 2000 & 2000 \\
Battery usage costs [€/kWh] & 0.35 & 0.30 \\
Auxiliary consumption [kW] & 4.5 & 4.5 \\
Maximum charging power [kW] & 48 & 60 \\
\hline
\end{tabular}

technical concepts differ significantly. Bus type 1 represents a lowweight depot charging battery bus with a total battery capacity of $90 \mathrm{kWh}$, like the Optare Metrocity [40]. In contrast, bus type 2 represents the BYD ebus and uses a battery with about $380 \mathrm{kWh}$, which is the maximum capacity offered on the market at this time [41]. The high battery capacity causes additional weight itself and requests mechanical reinforcements of the bus chassis, which leads to a further increase in weight. A direct consequence is expected for the energy consumption, and therefore for the energy efficiency for the offered transportation service.

The usable capacity of the battery fades over time due to aging effects. The end of life (EOL) is generally defined as a remaining capacity of $80 \%$ [42]. This buffer has to be taken into account in the vehicle scheduling to compute schedules that can be operated during the entire lifetime of a bus and its battery. Another option would be to use the entire battery capacity and to cope with a fading driving range of the bus. However, this would significantly increase the efforts in planning and handling of the buses and is therefore not desired by bus operators. A more detailed discussion of the battery design for public transport applications is provided in [43].

In addition to the energy buffer for battery aging, each bus should at any time have enough energy left to return to the depot. The usable energy $E_{\text {usable }}$ is defined as:

$E_{\text {usable }}=0,8 E_{\text {total,initial }}-e_{\text {con }} d_{\text {depot }}$.

Where $E_{\text {total,initial }}$ is the maximum battery capacity, $e_{\text {con }}$ is the energy consumption of an empty bus of $0.5 \mathrm{kWh} / \mathrm{km}$ for bus type 1 and $0.9 \mathrm{kWh} / \mathrm{km}$ for bus type 2 (determined by energy consumption simulation), and $d_{\text {depot }}$ is the maximum distance to the depot, $25 \mathrm{~km}$ in the present scenarios.

The assumed battery cost figures are based on estimates for the current market of electric bus batteries [44]. Slightly increased battery costs are defined for bus type 1 because the share of passive components, such as fuses, switches, and the battery management system, is higher than for the large battery of bus type 2 . The consideration of battery aging in this paper is reduced to cyclic aging in terms of equivalent full cycles (FCE); hence, further aging effects caused by temperature and depth of discharge are excluded from the scope of the study. The assumption of 2000 FCE has therefore been defined conservatively. The usage-dependent battery costs also cover additional expenses for replacements and a residual value at the EOL, since the cost calculation is based on linear depreciation.

The energy consumption per trip, for both service and deadheading trips, is determined by simulation in the preprocessing. Moreover, in addition to the energy demand of the traction system, the auxiliaries also have to be taken into account. This is done by including the average auxiliary consumption representing a summer day in midEurope with air conditioning for the driver. A load of $3 \mathrm{~kW}$ is assumed for the air compressor, steering support, passenger information, and 
Table 4

GGA parameters.

\begin{tabular}{ll}
\hline Parameter & Value \\
\hline Individuals per generation & 50 \\
Number of subpopulations & 4 \\
Crossover fraction & 0.2 \\
Migration interval [generations] & 20 \\
\hline
\end{tabular}

worst individuals from one generation are replaced by the best individuals from another. The usage of a greedy start and the absence of penalty functions lead to relatively good starting solutions. The largest improvements are achieved in the beginning by utilizing the large diversity of the generation. The decreasing diversity over the number of generations is caused by the crossover: continuous combining of the individual characteristics leads to reduced differences between individuals.

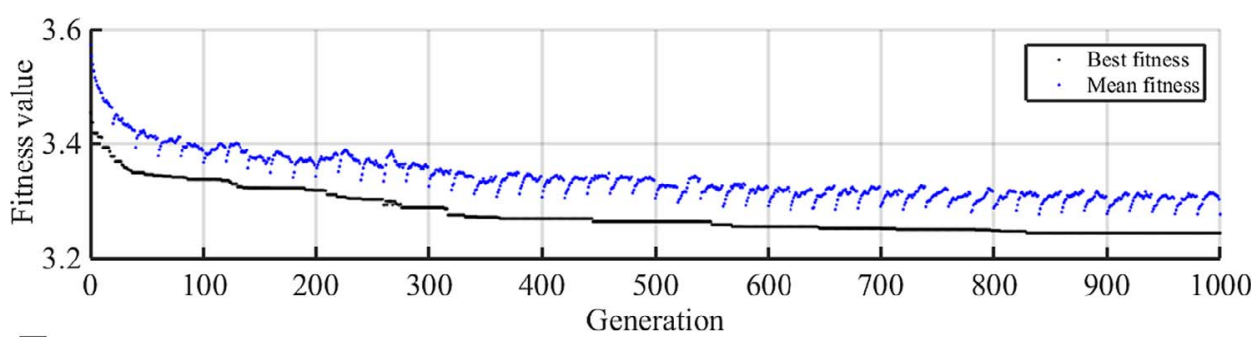

Fig. 10. Progress of the algorithm in scenario A for a homogeneous fleet of bus type 1 , illustrated by the fitness value in $€ / \mathrm{km}$ service trip operation and the relative diversity.

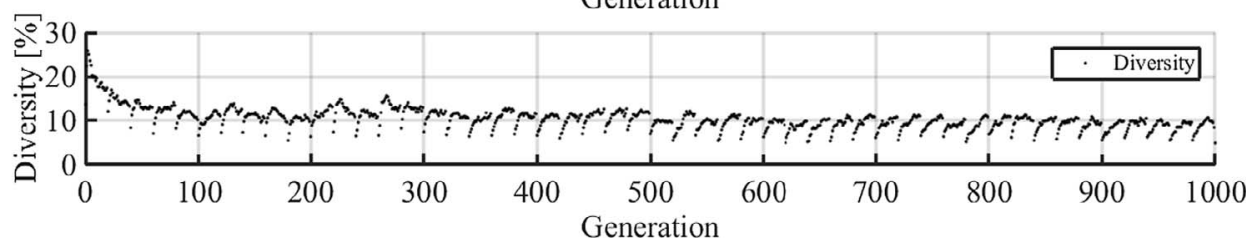

lighting, and $1.5 \mathrm{~kW}$ is assumed for the driver air conditioning [45]. The efficiencies of the motor (0.90), inverter (0.95), and batteries (0.96) are considered to be identical for both bus types. It is furthermore assumed that both bus types can be charged with the same charger. The defined charging power represents the charging capabilities of the Optare Metrocity and BYD ebus.

\subsection{Genetic algorithm settings and performance}

The GGA is run in the case study with the parameter set shown in Table 4. These parameters have been selected after multiple experiments with scenarios A and B. Especially the usage of multiple subpopulations is beneficial for the stability of the algorithm.

Fig. 10 shows the progress of the algorithm for scenario A. The upper graph highlights the fitness value of the best solution found so far and the mean value of all individuals in one generation. The diversity in the lower part is measured block wise and given as a percentage. A value of $100 \%$ indicates that all blocks (trip sequences) in the entire generation differ from each other, whereas a diversity value close to $0 \%$ represents only identical blocks. A definition of the diversity individualwise would lead to higher values.

The periodic interferences in the diversity and the mean fitness value, visible in Fig. 10, are caused by the migration during which the

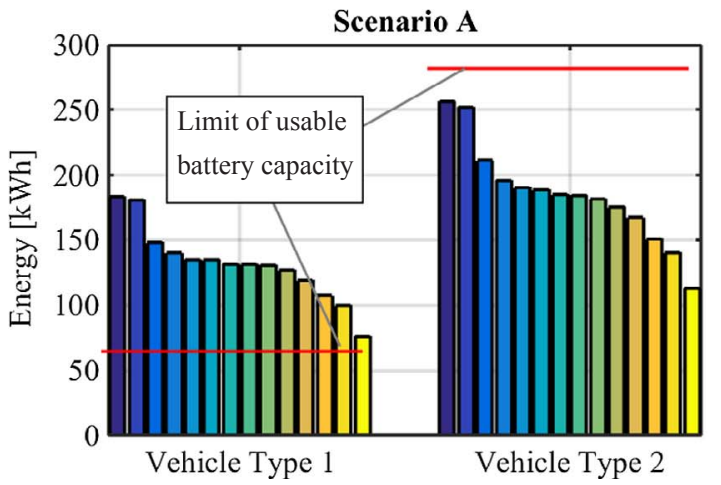

\subsection{Results for homogeneous and heterogeneous fleet composition}

The EVS-FMC is solved for both homogeneous fleets (either bus type 1 or 2), and for the heterogeneous fleet (possibility of choosing both bus types). The current diesel bus operating plan, as provided by the operator, serves as a reference for the quality of the EVS-FMC. This is the best lower bound available, as the optimal solution for the EVS-FMC is unknown and the problem itself is intractable to solve directly. The diesel bus solution provides a lower bound on the fleet size, deadhead mileage, and operation time.

An efficient electrification at minimal operational effort could be achieved by a one-by-one replacement. In this case, a diesel bus would be replaced by an electric bus without changing the operational scheme. However, the limited battery capacity would restrict this process in most cases.

The energy demand of the original diesel bus runs has been calculated based on the technical parameters of both bus types. The results provided in Fig. 11 reveal the increased energy consumption of bus type 2 , which is 40 to $50 \%$ higher than that of bus type 1 . The main reason for this is the large battery capacity causing an increased weight of bus type 2. On the one hand, bus type 2 therefore has a clear drawback regarding energy efficiency, since both bus types offer the same passenger capacity. On the other hand, only bus type 2 is able to operate

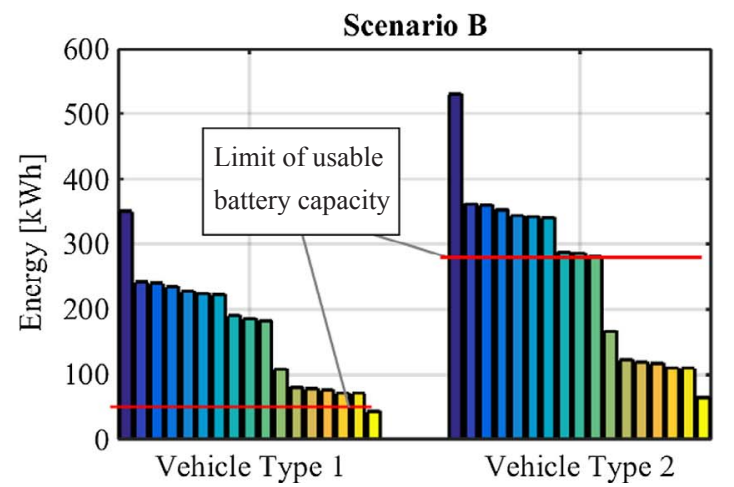

Fig. 11. Energy demand of the original bus runs and usable battery capacity limits of both bus types in scenarios A and B. Bus runs are separated by color scheme. 
the original diesel bus runs in scenario A. This is indicated by the red horizontal line representing the maximum usable battery capacity for both bus types ( $60 \mathrm{kWh}$ for bus type 1 and $282 \mathrm{kWh}$ for bus type 2). It is clearly visible that the usable battery capacity of bus type 1 is insufficient for almost every diesel bus run analyzed. The same applies for bus type 2 in scenario B. Therefore, vehicle schedule adaptations are required, which could potentially result in additional vehicles, deadhead mileage, and driver expenses.

\subsubsection{Scenario $A$}

The results of scenario A are shown in Table 5. The objective is provided per service kilometer and as a total cost figure covering the entire planning horizon of 12 years' continuous operation. As expected from the overview in Fig. 11, the usage of bus type 1 leads to an increased fleet size, whereas a homogeneous fleet with type 2 buses matches the conventional fleet size of 14 buses. However, although the fleet sizes are identical, there are differences in the number of blocks, deadhead mileage, and bus runtime. As indicated by the additional block, one bus visits the depot after the morning operation. Due to this and further improvements of the schedule, the deadhead mileage is increased, whereas the bus runtime decreases. It has to be stated here that the diesel reference scenario is optimized manually by the operator. The direct replacement of all diesel buses with type 2 buses, as is possible for scenario $A$, would increase cost by over 1 million $€$ in comparison to the minimum cost solution to the EVS-FMC. This would result in a TCO (investment and operational costs in 12 years) of 32.24 million $€$, thus indicating the value of solving the EVS-FMC.

The mixed fleet solution uses a total of 14 buses as well. The fleet is composed of six buses of type 1 and eight buses of type 2 . Similar to the homogeneous fleet of bus type 1, only three chargers are required, instead of the four utilized for a homogeneous fleet of type 2 vehicles. The usage of the lightweight buses with the small battery capacity causes that a high number of buses have to return to the depot during the daytime. This increases the deadhead mileage in comparison to using only vehicles of type 2 , but also enables the minimization of the number of chargers.

The variation in individual measures, such as fleet size, deadhead mileage, and energy consumption, is larger than in the TCO. This is caused by the compensation of different cost figures. The low weight of bus type 1 enables a reduced energy consumption compared to bus type 2 , but this advantage is partly compensated by the higher battery usage costs of bus type 1 . Additional expenses for the purchasing of bus type 2 are furthermore mitigated by the exploitation of fewer buses. Within the mixed fleet approach, on the other hand, the additional degrees of freedom are used to find a tradeoff between the competing cost figures. However, the savings are only minor, so that bus operators may stick to only one bus type to reduce efforts in depot and workshop. In this case, bus type 2 would be the preferred solution.

Table 5

Results for scenario A compared to the diesel bus scenario.

\begin{tabular}{lllll}
\hline SCENARIO A & $\begin{array}{l}\text { Diesel } \\
\text { reference }\end{array}$ & Bus type 1 & Bus type 2 & Mixed \\
\hline Fleet size & 14 & 17 & 14 & $\begin{array}{l}14 \text { (6 VT1, 8 } \\
\text { VT2) }\end{array}$ \\
Number of blocks & 14 & 38 & 15 & 22 \\
Number of chargers & - & 3 & 4 & 3 \\
Deadhead mileage [km] & 254 & 527 & 397 & 462 \\
Bus runtime [h] & 189 & 197 & 182 & 186 \\
Electricity consumption & - & 1.99 & 2.72 & 2.48 \\
$\quad[$ MWh] & - & 3.2438 & 3.2876 & 3.2207 \\
TCO [€/km] & - & 31.23 & 31.65 & 31.01 \\
TCO [total million $€$ ] & - & &
\end{tabular}

Table 6

Results for scenario B compared to the diesel bus scenario.

\begin{tabular}{lllll}
\hline SCENARIO B & $\begin{array}{l}\text { Diesel } \\
\text { reference }\end{array}$ & Bus type 1 & Bus type 2 & Mixed \\
\hline Fleet size & 12 & 18 & 13 & $\begin{array}{l}15(9 \text { VT1, } 6 \\
\text { VT2) }\end{array}$ \\
Number of blocks & 17 & 73 & 27 & 48 \\
Number of chargers & - & 5 & 5 & 4 \\
Deadhead mileage [km] & 363 & 590 & 409 & 499 \\
Bus runtime [h] & 160 & 187 & 163 & 174 \\
Electricity consumption & - & 2.94 & 4.34 & 3.65 \\
$\quad$ [MWh] & - & 2.4736 & 2.4830 & 2.4567 \\
TCO [€/km] & - & 33.01 & 33.14 & 32.79 \\
TCO [total million $€$ ] & - & &
\end{tabular}

\subsubsection{Scenario $B$}

In Table 6, the TCO for a homogeneous fleet of type 1 or 2 buses, and the TCO for a heterogeneous fleet are relatively close. As expected, more vehicles of type 1 are needed than diesel buses or buses of type 2 in the homogeneous scenario. Furthermore, the deadhead mileage increases by $62 \%$ to $590 \mathrm{~km}$ in comparison to the diesel buses, and by $44 \%$ in comparison to the $409 \mathrm{~km}$ deadhead mileage for a homogeneous fleet of type 2 buses. Thus, the homogeneous type 1 fleet results in higher costs of drivers in comparison to the type 2 vehicles. On the other hand, although the deadhead mileage is higher, the $32 \%$ lower energy consumption of bus type 1 (2.94 vs $4.34 \mathrm{MWh}$ ) offers large savings compared to bus type 2 . The difference of $1.4 \mathrm{MWh}$ per day yields cost benefits in the range of $0.11 € / \mathrm{km}$, thus leading to slightly lower costs for a homogeneous type 1 fleet than for a homogeneous type 2 fleet (TCO of 33.01 vs 33.14 million $€$ ).

The usage of a mixed fleet enables a cost reduction of $220,000 €$ within the planning horizon of 12 years in comparison to a homogeneous fleet of type 1 vehicles. The mixed fleet is advantageous because of the increased vehicle requirements during peak hours. The vehicles of type 1 are mainly used during peak hours, while type 2 buses cover the base demand, thus saving $15 \%$ of deadheading distance and $7 \%$ of bus run time in comparison to the solution of a homogeneous type 1 fleet.

The computed results enable the bus operator to decide on a depot charging battery bus concept. An important factor in this process is the local situation at the bus depot. Depending on the available space, it could be beneficial to minimize the number of buses. Therefore, a homogeneous fleet of type 2 buses could be advantageous despite their additional costs. If this issue is uncritical, the advancements of a mixed fleet (reduced costs and charger demand) should be utilized.

\subsubsection{Evaluation of charger optimization}

The usage of the MILP formulation enables the GGA to evaluate each individual based on full information. As described in Section 3.2.2, an alternative strategy would be to neglect charger costs during the GGA runtime and conduct the rescheduling of charging events afterwards. Table 7 shows the best results produced by the GGA without considering charger costs during the runtime (charger optimization is conducted subsequently) compared to the solution with charger optimization during runtime for scenario A.

A subsequent charger optimization determines that a total of four chargers are required, which means that one additional charger is used compared to the reference. The objective for this solution is $3.2380 € /$ $\mathrm{km}$, or 31.18 million $€$ for the entire planning horizon. The cost increase of $170,000 €$ compared to the reference with activated charger optimization even exceeds the expenses for the additional charger. The major cost variation is caused by the different fleet composition. The results indicate that the usage of the charger optimization within the fitness function supports the exploration of bus type 1 . This could be caused by the ability of bus type 1 to reduce the number of chargers by shifting charging events from the night to the daytime. 
Table 7

Results for scenario A for a mixed fleet with and without charger optimization during runtime.

\begin{tabular}{lll}
\hline SCENARIO A & $\begin{array}{l}\text { Mixed fleet with charger } \\
\text { optimization }\end{array}$ & $\begin{array}{l}\text { Mixed fleet without } \\
\text { charger optimization }\end{array}$ \\
\hline Fleet size & $14(6 \mathrm{VT} 1,8 \mathrm{VT} 2)$ & 14 (5 VT1, 9 VT2) \\
Number of blocks & 22 & 20 \\
Number of chargers & 3 & 4 \\
Deadhead mileage $[\mathrm{km}]$ & 462 & 458 \\
Bus runtime $[\mathrm{h}]$ & 186 & 186 \\
Electricity consumption & 2.48 & 2.53 \\
$\quad$ & & \\
TCO $[€ / \mathrm{km}]$ & 3.2207 & 3.2380 \\
TCO [total million $€$ ] & 31.01 & 31.18 \\
\hline
\end{tabular}

The evaluation has also been conducted for scenario B. In contrast to the results of scenario A, the best solution found without charger optimization in this case equals the solution with activated charger optimization.

\section{Conclusions}

This paper has introduced the EVS-FMC and addressed the upcoming issues in the transformation of public transport diesel bus fleets to electric bus fleets. The solution approach is based on a GGA in combination with a MILP formulation. It aims to minimize the TCO, consisting of vehicle investment, charger investment, operational costs, and energy expenses.

The approach was applied within a case study of real-world electrification scenarios of two different cities. Homogeneous and heterogeneous fleets were composed out of two electric bus types, one based on a lightweight bus with a strictly limited range and the other on a high range bus. The results showed that in both scenarios, the lightweight bus offers the more energy efficient mode of transportation, although the deadheading mileage increases. The energy consumption savings range from $27 \%$ in scenario A to $32 \%$ in scenario B. However, the limited range requires additional vehicles, and several charging phases throughout the day lead to additional driver costs due to deadheading. The TCOs of both bus types are therefore relatively close, revealing a compensation by the additional costs of the lightweight bus for vehicles and drivers for reduced energy costs. An operation of a mixed fleet of both bus types can reduce the TCO, depending on the characteristics of the bus route. The TCO of a mixed fleet operation utilizing both bus types was calculated as $3.22 € / \mathrm{km}$ in scenario A and $2.45 € / \mathrm{km}$ in scenario B. Although additional technical and economical parameters were utilized in the scenarios, the TCO difference is significant, which underlines the strong dependency of the operational characteristics. Especially bus routes with an increased peak vehicle demand seem to be beneficial for the operation of depot charging battery buses.

The proposed methodology offers a wide range of applications, the most suitable being within feasibility studies to evaluate electrification options for bus networks. In contrast to existing approaches, which only focus on the technical feasibility on the basis of the original diesel bus runs, the EVS-FMC enables a joint technical and operational analysis. Adjustments of vehicle schedules are considered and monetized within a TCO calculation so that a reasonable balance of charging infrastructure investments and operational adjustments can be determined. The presented approach has been integrated into the software toolbox of ebusplan (ebusplan GmbH, Germany) and is currently utilized in consultancy projects ranging from less than 10 to more than 200 buses.

\section{Acknowledgements}

This work was conducted as part of the Climate-KIC research project LoCarUT, funded by the European Institute of Innovation and Technology (EIT).

\section{References}

[1] Wang R, Wu Y, Ke W, Zhang S, Zhou B, Hao J. Can propulsion and fuel diversity for the bus fleet achieve the win-win strategy of energy conservation and environmental protection? Appl Energy 2015;147:92-103.

[2] Wang E, Di Guo, Yang F. System design and energetic characterization of a fourwheel-driven series-parallel hybrid electric powertrain for heavy-duty applications. Energy Convers Manage 2015;106:1264-75.

[3] Sen B, Ercan T, Tatari O. Does a battery-electric truck make a difference?: - Life cycle emissions, costs, and externality analysis of alternative fuel-powered Class 8 heavy-duty trucks in the United States. J Clean Prod 2017;141:110-21.

[4] Wesseling JH. Explaining variance in national electric vehicle policies. Environ Innov Societal Transit 2016.

[5] Hao H, Ou X, Du J, Wang H, Ouyang M. China's electric vehicle subsidy scheme: Rationale and impacts. Energy Policy 2014;73:722-32.

[6] Li J-Q. Battery-electric transit bus developments and operations: a review. Int J Sustain Transport 2014;10(3):157-69.

[7] Nykvist B, Nilsson M. Rapidly falling costs of battery packs for electric vehicles. Nat Clim Change 2015;5(4):329-32.

[8] Hoff A, Andersson H, Christiansen M, Hasle G, Løkketangen A. Industrial aspects and literature survey: Fleet composition and routing. Comput Oper Res 2010;37(12):2041-61.

[9] Pelletier S, Jabali O, Laporte G. 50th anniversary invited article-goods distribution with electric vehicles: review and research perspectives. Transport Sci 2016;50(1):3-22.

[10] Goeke D, Schneider M. Routing a mixed fleet of electric and conventional vehicles. Eur J Oper Res 2015;245(1):81-99.

[11] Lebeau P, de Cauwer C, van Mierlo J, Macharis C, Verbeke W, Coosemans T. Conventional, hybrid, or electric vehicles: which technology for an urban distribution centre? Sci World J 2015;2015:302867.

[12] van Duin JHR, Tavasszy LA, Quak HJ. Towards E(lectric)- urban freight: first promising steps in the electric vehicle revolution. Eur Transport Trasporti Europei 2013;54:9.

[13] Gonçalves F, Sónia R Cardoso, Susana Relvas, Barbosa-Póvoa APFD. Optimization of a distribution network using electric vehicles: A VRP problem. In: the Proceedings of the IO2011-15 Congresso da associação Portuguesa de Investigação Operacional, Coimbra, Portugal 2011:18-20.

[14] Hiermann G, Puchinger J, Ropke S, Hartl RF. The electric fleet size and mix vehicle routing problem with time windows and recharging stations. Eur J Oper Res 2016;252(3):995-1018.

[15] Sassi O, Cherif-Khettaf WR, Oulamara A. Vehicle routing problem with mixed fleet of conventional and heterogenous electric vehicles and time dependent charging costs. Int J Math, Comput, Phys, Electr Comp Eng 2015;3:171-81.

[16] Li J-Q. Transit bus scheduling with limited energy. Transport Sci 2014;48(4):521-39.

[17] Wang H, Shen J. Heuristic approaches for solving transit vehicle scheduling problem with route and fueling time constraints. Appl Math Comput 2007;190(2):1237-49.

[18] Hao X, Jin W, Wei M. Max-Min ant system for bus transit multi-depot vehicle scheduling problem with route time constraints. In: 2012 10th World Congress on Intelligent Control and Automation (WCICA 2012); 2012, p. 555-60.

[19] Paul T, Yamada H. Operation and charging scheduling of electric buses in a city bus route network. In: 2014 IEEE 17th International Conference on Intelligent Transportation Systems (ITSC); 2014: p. 2780-6.

[20] Chao Z, Xiaohong C. Optimizing battery electric bus transit vehicle scheduling with battery exchanging: model and case study. Proc - Soc Behav Sci 2013;96:2725-36.

[21] Ke B-R, Chung C-Y, Chen Y-C. Minimizing the costs of constructing an all plug-in electric bus transportation system: a case study in Penghu. Appl Energy 2016;177:649-60.

[22] Wen M, Linde E, Ropke S, Mirchandani P, Larsen A. An adaptive large neighborhood search heuristic for the Electric Vehicle Scheduling Problem. Comput Oper Res 2016;76:73-83.

[23] Ercan T, Zhao Y, Tatari O, Pazour JA. Optimization of transit bus fleet's life cycle assessment impacts with alternative fuel options. Energy 2015;93:323-34.

[24] Xu Y, Gbologah FE, Lee D-Y, Liu H, Rodgers MO, Guensler RL. Assessment of alternative fuel and powertrain transit bus options using real-world operations data: Life-cycle fuel and emissions modeling. Appl Energy 2015;154:143-59.

[25] Ostadi A, Kazerani M. A comparative analysis of optimal sizing of battery-only, ultracapacitor-only, and battery-ultracapacitor hybrid energy storage systems for a city bus. IEEE Trans Veh Technol 2015;64(10):4449-60.

[26] Kukkonen S, Erkkila V, Manninen A, Haavisto J, Pihlatie M. Method for dimensioning battery and thermal management systems for heavy-duty vehicle applications using aged battery experimental data and advanced modelling techniques. ECS Trans 2015;68(2):83-95.

[27] Chen J, Atasoy B, Robenek T, Bierlaire M, Thémans M. Planning of feeding station installment for electric urban public mass-transportation system. In: 13th Swiss Transportation Research Conference; 2013. 
[28] Kunith A, Mendelevitch R, Goehlich D. Electrification of a city bus network-an optimization model for cost-effective placing of charging infrastructure and battery sizing of fast-charging electric bus systems. Int J Sustain Transport 2017;11(10):707-20.

[29] Olsson O, Grauers A, Pettersson S. Method to analyze cost effectiveness of different electric bus systems. In: Proceedings of EVS29 International Battery, Hybrid and Fuel Cell Electric Vehicle Symposium 2016:1-12.

[30] Falkenauer E. Genetic algorithms and grouping problems. Chichester: Wiley; 1998.

[31] Pankratz G. A grouping genetic algorithm for the pickup and delivery problem with time windows. OR Spectrum 2005;27(1):21-41.

[32] Hosny MI, Mumford CL. Investigating genetic algorithms for solving the multiple vehicle pickup and delivery problem with time windows. In: Proceedings of the VIII Metaheuristics International Conference MIC 2009.

[33] Grande L, Paillard E, Hassoun J, Park J-B, Lee Y-J, Sun Y-K, et al. The lithium/air battery: still an emerging system or a practical reality? Adv Mater (Deerfield Beach, Fla.) 2015;27(5):784-800.

[34] Veneri O, Capasso C, Patalano S. Experimental study on the performance of a ZEBRA battery based propulsion system for urban commercial vehicles. Appl Energy 2017;185:2005-18.

[35] Sinhuber P, Rohlfs W, Sauer DU. Study on power and energy demand for sizing the energy storage systems for electrified local public transport buses. In: 2012 IEEE Vehicle Power and Propulsion Conference (VPPC); 2012, p. 315-20.

[36] Rogge M, Wollny S, Sauer D. Fast charging battery buses for the electrification of urban public transport-a feasibility study focusing on charging infrastructure and energy storage requirements. Energies 2015;8(5):4587-606.

[37] MATLAB. The MathWorks. Inc, Natick, Massachusetts, United States 2013;488.

[38] CPLEX IIM. V12. 1: User's Manual for CPLEX. International Business Machines Corporation 2009;46(53):157.

[39] Lindgren L. Full electrification of Lund city bus traffic - A simulation study: Department of Industrial Electrical Engineering and Automation, Lund Institute of Technology; 2016; Available from: http://portal.research.lu.se/portal/files/ 5590392/7362616.pdf.

[40] Optare Group Ltd. Specification of Optare Electric Vehicles; 2016; Available from: http://www.optare.com/s/Optare-electric-vehicles-brochure-xd0x.pdf. [July 22, 2016].

[41] BYD Europe B.V. Specification of BYD EBUS-12; 2015; Available from: http:// bydeurope.com/downloads/eubs_specification/BYD_12_Meters_Electric_bus.pdf. [July 22, 2016].

[42] Vetter J, Novák P, Wagner MR, Veit C, Möller K-C, Besenhard JO, et al. Ageing mechanisms in lithium-ion batteries. J Power Sources 2005;147(1-2):269-81.

[43] Rothgang S, Rogge M, Becker J, Sauer D. Battery design for successful electrification in public transport. Energies 2015;8(7):6715-37.

[44] California Air Resources Board. Advanced Clean Transit Battery Cost for HeavyDuty Electric Vehicles (Discussion Draft). [August 12, 2017]; Available from: https://www.arb.ca.gov/msprog/bus/battery_cost.pdf.

[45] Eberspächer. Driver's seat air conditioning - Product Datasheet AC 403 E G2. [August 07, 2017]; Available from: https://www eberspaecher.com/en/products/ air-conditioning/bus-ac-solutions/products/drivers-seat-air-conditioning.html. 